\title{
A Versatile IoT-Approach to Process Data Acquisition
}

\author{
Bernd-Arno Behrens, Richard Krimm, Jonas Koß and André Höber
}

Bernd-Arno Behrens. Leibniz University Hannover, Institute of Forming Technology and Machines, An der Universität 2, 30823

Garbsen, Germany

Richard Krimm. Leibniz University Hannover, Institute of Forming Technology and Machines, An der Universität 2, 30823

Garbsen, Germany

Jonas Koß. Leibniz University Hannover, Institute of Forming Technology and Machines, An der Universität 2, 30823 Garbsen,

Germany

André Höber. Leibniz University Hannover, Institute of Forming Technology and Machines, An der Universität 2, 30823 Garbsen, Germany

Corresponding author: hoeber@ifum.uni-hannover.de

Abstract. The acquisition and evaluation of process data in production engineering holds great potential. This allows detecting faulty processes at an early stage and processes to be optimized even more efficiently. However, the use of technologies for data acquisition in the manufacturing industry is far less widespread than the mentioned potential implies. This paper presents an Internet of Things approach by means of which production environments can be retrofitted easily and cost-effectively.

Keywords. Data Acquisition, Internet of Things, Production Engineering

\section{Introduction}

Big Data Analytics have been used in online marketing for some time now to control campaigns in a targeted manner and to track the consumption behavior of customers [1]. Although data analysis in production environments is generally also considered to have a great influence on the success of a company [2], it is less widely used than this implies. Especially component-related process data acquisition offers potentials that remain widely unused so far.

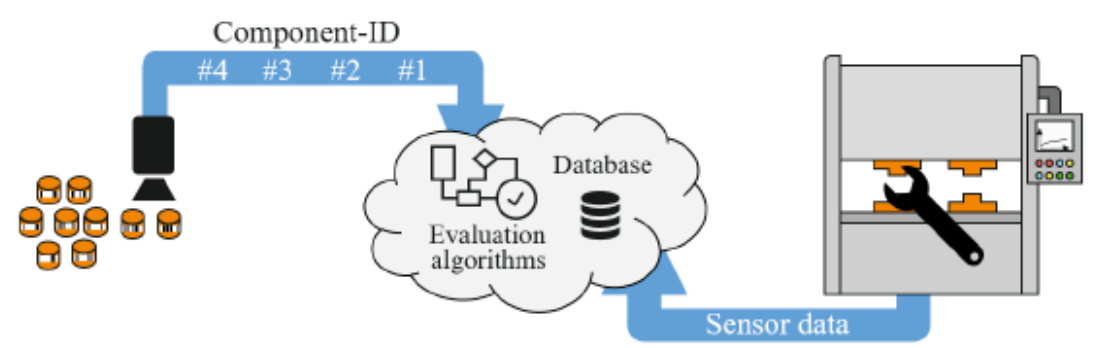

Fig. 1: Component-related process data acquisition

First approaches are aiming to use the existing digital infrastructures in companies' production facilities to collect data from collaborating machines, which is in accordance with the basic idea of the Internet of Things (IoT). Such concepts can be applied, for example, in car body manufacturing plants [3]. Based on prior measurements and quality inspection, an estimation of expected faulty parts for further production batches can be made. The long-term storage of process data while simultaneously assigning the data to a specific component (Fig. 1) enables systematic analysis of the causes for customer complaints and increases the potential for the optimization of processes. 
According to studies, the reasons for the poor use of such applications are in many cases a lack of will, doubts regarding the benefits or difficulties in implementing them [4]. Furthermore, solutions available on the market often only allow the acquisition of machine data from specific data sources (e.g. PROFIBUS network controller) and are only of limited use in production environments characterized by a variety of communication and sensor technologies.

At the same time, the growing market of the Internet of Things, also in the consumer sector, has led to a constantly growing number of providers of suitable hardware and software. This offers the potential to create simple and costeffective solutions tailored to specific requirements [5].

In this paper, an IoT approach is presented which allows collecting data from several data sources adapted to the needs of production engineering, especially forming technology. To achieve the best performance possible, the choice of components and appropriate communication systems is of great importance and has to be done with great care. The aim is not only to develop a flexible system to be implemented in a wide range of production environments, but also to provide cost-effectiveness by using only highly functional components. For this purpose, the various solutions that are considered are compared with each other and combined in a suitable way. The system presented here allows comprehensive data acquisition with subsequent storage in a cloud database and thus contributes to the development of a cost-efficient smart factory.

\section{State of Technology}

\subsection{Process Data and Data Sources in Forming machines}

The most intuitive way to collect data probably is reading out data from the press control. However, this would lead to considerable costs for control changes. In addition, there are relevant process parameters that are inaccessible via the press control or at least not obtainable in the required resolution, e.g. machine vibrations. In addition, demanding production processes are monitored with additional, complex measurement technology for quality assurance purposes [6]. On the other hand, there are parameters such as the motor torque that cannot be monitored with retrofitted sensors or where intervention in the press system would lead to a loss of warranty. In many cases the use of both, internal and external sensors is useful for efficient data acquisition. In order to assign process data properly, parameters such as machine settings are also important. These parameters do not change during the course of a batch and therefore do not have to be monitored continuously. The three groups of different process data are summarized in Fig. 2.

\begin{tabular}{|l|}
\hline Batchwisely Measured \\
\hline e.g. Machine Settings \\
- Stroke Rate \\
- Stroke \\
- Feeding Path \\
\hline
\end{tabular}

\begin{tabular}{|l|}
\hline Press Control \\
\hline - Motor Current \\
- Motor Torque \\
- Motor Temperature \\
\hline
\end{tabular}

Additional Sensors
- Ram Position
- Process Force
- Machine Vibrations

Derived Parimeters
From Measured Parameters
- Snap-Through Shock
(Magnitude, Frequency)
- Wear And Tear

Fig. 2: Categories of different process-related data

When designing a data acquisition system that also accesses process data available in the press control, several challenges need to be faced. Since the purchase of a forming machine is a great financial investment, the machines are often used for several decades. As a result, many small and medium-sized companies have inventory plants that do not necessarily represent the latest state of technology. Due to the wide range of the age of presses in many production facilities, the control systems of the machines show a great variety regarding the sensor and communication 
technology used.

In modern press control systems, communication is based on Industrial Ethernet systems such as EtherCAT, PROFINET or EthernetIP. The components of older press systems usually communicate using field bus systems such as PROFIBUS or CANopen. Although Industrial Ethernet is replacing fieldbus systems step by step, fieldbuses are still widely in use with a market share of about one third [7].

\subsection{Available Solutions}

Modern control systems already allow the establishment of wireless connections to other machines, in order to exchange data. However, the technology still used in production environments is often older and does not offer such functions. Further, considering an extensive data acquisition, additional sensors may be required. Applying these sensors to the machine control system can be time-consuming and costly. However, even this is not always practical, if the monitored data is required in a higher resolution than offered by the machine control system. Therefore, in many cases, a flexible retrofit solution is of high interest.

Currently several solutions exist on the market that allow the acquisition and storage of sensor data. One of the marketleading systems is Kistler's KiDAQ [8]. KiDAQ is a modular system that can be adapted to the specific requirements of the measurement task by selecting specific sensor modules. Various modules are available for connecting analogous sensors (e.g. LASER distance sensors, thermocouples) which provide high resolution (up to 24 bit) at high sampling rates (up to $100 \mathrm{kS} / \mathrm{s}$ ). However, fieldbus interfaces to be integrated in machine networks are not available. A wireless transmission of the incoming data is not possible either. In addition, the system is linked to the use of the KiStudio Lab software. A free programming for data processing and storage in other possibly already existing database structures is not possible.

Another available system is the NI CompactDAQ [9]. Similar to the KiDAQ it is a modular system based on a controller that can be extended with specific input modules. In addition, a module is offered which allows communication with CAN bus systems. Other bus protocols are not supported. In order to use the system, the software LABView is required. This not only limits user access, but also requires the purchase of a license.

Another available solution, which already meets the requirements of the production environment, is the ip4Cloud/ SEC3PB by IPCOMM [10]. The controller is compact and can be integrated in PROFIBUS systems. The system also provides integrated interfaces for cloud and database connection. However, further external sensors cannot be integrated easily. Also, no integration in Industrial Ethernet systems is possible.

Apart from that, the increasing demand for smart home applications in the private sector accelerated the availability of low-cost open source solutions for Internet of Things applications. Based on this, commercial solutions have already been developed, which can be used for the purpose of data acquisition. Two examples are the netPI by Hilscher Automation [11] and the RevPi series by KUNBUS [12]. Both systems are based on the Raspberry Pi board.

The RevPi is intended as a compact control system in the first place, but can also be used as a data acquisition system. It has a modular design with a wide variety of functionalities and is similar in structure to the KiDAQ and CompactDAQ. In addition, various gateway modules are available, which provide an interface to Industrial Ethernet systems such as PROFINET [13], EtherNet/IP, POWERLINK or even field bus systems such as PROFIBUS [14] and CANopen [15]. The system is freely programmable and can therefore be adapted to other applications. Wireless transmission to serverlocated database structures is also possible. A huge disadvantage is the long minimum cycle time of $5 \mathrm{~ms}$, which limits the system with regard to data acquisition.

The netPI has a real-time network controller and is not limited by excessive cycle time. The netPI is a compact 
controller, which is multi-protocol compatible and can be used as Industrial Ethernet Slave. The necessary protocol stacks are pre-installed. Additional modules are not necessary. In order to use the system in a PROFIBUS network, an additional adapter is required. The netPI is freely programmable and enables customized processing and wireless data transmission. However, the connection of external sensors is not possible directly.

Both solutions demonstrate that customized solutions can also be developed based on commercially available microcontrollers. The best known probably are the Arduino and the Raspberry Pi boards. The IoT-board Arduino Yùn Rev 2 for example is equipped with an integrated analogue-digital converter, a second Linux processor to enhance functionality and it can be used for wired and wireless LAN connections. The variety of interfaces and expansion modules allow them to be used in many different applications [16]. In addition to a wired and wireless LAN interface, the Raspberry Pi board features a CSI camera interface and an SPI bus connector for further enhancement of functionality [17]. As of today, a single solution meeting all the requirements of modern production environments is not yet available.

\section{Development of a Data Acquisition System}

\subsection{Concept for Data Acquisition}

The work described in this paper aims at developing a solution to component-related process data acquisition that can be used and retrofitted as flexibly as possible and is cost-effective. In this case, flexible use means that the data aggregator must be able to receive data from as many different data sources as possible. As already mentioned, there are parameter values, which can only be obtained from the press communication network and others, which can only be obtained by external sensor technology. The data aggregator must therefore provide Industrial Ethernet and field bus communication as well as connectivity to external sensors. Analogous signals should be acquired with high resolution (16 bit) and be sampled quickly ( $>1 \mathrm{kHz}$ ). In addition, component identification marks are to be captured. One approach could be to equip the workpiece material with magnetic identification markers [18]. However, this method would be very time consuming and expensive. Hence, in this case, QR codes will be applied to the material for testing. In order to scan these codes, the data acquisition system requires an interface for a camera system.

After the process data and the component identification marks are captured, the data must be transmitted to a database server wirelessly. The data acquisition system thus needs to have a WLAN interface and be freely programmable so that, for example, functions for implementing the communication protocol MQTT can be used. The MQTT protocol uses three entities: Publisher, Broker and Subscriber. Publishers are connected to the Broker and send data for a certain Topic. Subscribers are also connected to the Broker, but receive data on a specific Topic. The Broker manages the incoming data from the Publisher and assigns them to the according Topic. It also routes the data packets on a Topic to the Subscribers in the network [19]. Since 2013, MQTT is the standardized protocol for IoT applications.

The concept for the component-related data acquisition system adapted to the needs of production environments is shown in Fig. 3. 


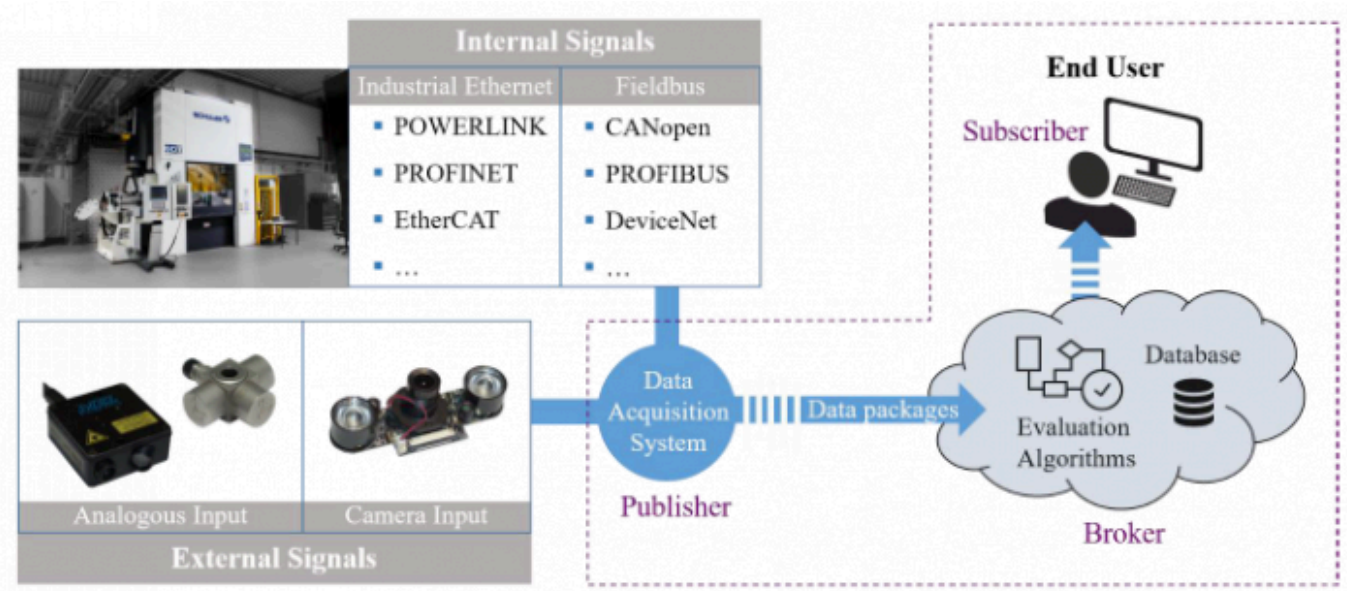

Fig. 3: Concept for component-related process data acquisition

\subsection{Setup}

As the previous sections depict, there is a wide range of solutions. Since they do not meet all needs, a specific combination of several components is required. In the introduction, the implementation costs were mentioned as one reason for the reluctance of the industry. Hence, one aim of the presented work is to provide a cost-effective system that is easy to apply and retrofit. Therefore, it was initially considered to develop a solution based on a single microcontroller. A significant difficulty was the interface to Industrial Ethernet and Fieldbus systems, both in terms of software and hardware.

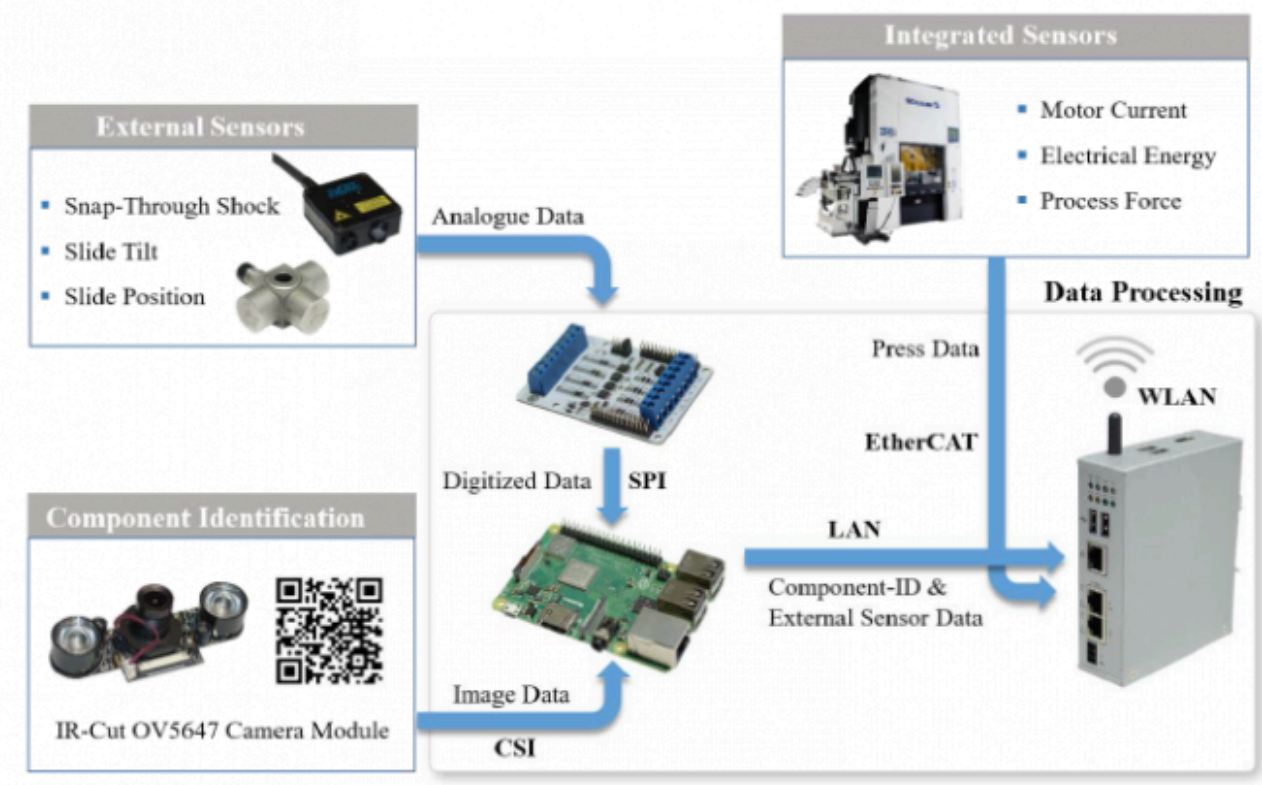

Fig. 4: Data acquisition system

The netPI RTE 3 was chosen as the main component of the data acquisition system. It is based on the Raspberry Pi 3 
model B and additionally equipped with a multi-protocol network controller. However, since there is no possibility to read out analogous signals, an additional device has to be connected to the netPI. In this case, an additional Raspberry Pi 3 Model B+ was chosen for reasons of compatibility. It provides necessary interfaces to enhance functionality, e.g. boards can be connected for the acquisition of sensor signals. The additional sensor board is desired to have the highest sampling rate and resolution possible. The research led to Widgetlords Pi-SPi-8AI-16B analogue input interface [20]. The sensor board is equipped with a 16-bit ADC and 8 inputs with a sampling rate of $500 \mathrm{kS} / \mathrm{s}$. Each four inputs are designed for voltage signals up to 12 VDC and current signals between 4-20 mA. In addition, the Raspberry Pi has a camera interface (CSI). The infrared camera IR-Cut OV5647 [21] with light sources was chosen for reading the identification features such as QR codes. This camera provides evaluable images even under unfavorable exposure conditions in a production environment. The setup of the entire data acquisition system is illustrated in Fig. 4.

The central component of the data acquisition system is the netPI RTE 3, in which incoming data are merged and forwarded to the database server via WLAN. Some of the data originate from the control system of a servo press available at the IFUM. For this purpose, the netPI has to be integrated into the press control system as an EtherCAT slave. The camera signals as well as the sensor values of the external sensors are merged on the Raspberry Pi and then transmitted to the netPI via LAN interface. The data acquisition system finally sends the process data to the database server. On the server, the MQTT-Broker distribution Apache ActiveMQ is installed. This software has a plug-in by means of which the incoming data can be stored in the Influx database. The influxdb is a database management system for storing chronological data.

In the context of the MQTT protocol, the data acquisition system represents the publisher, the database server including the database of the broker while the user acts as the subscriber (Fig. 5).

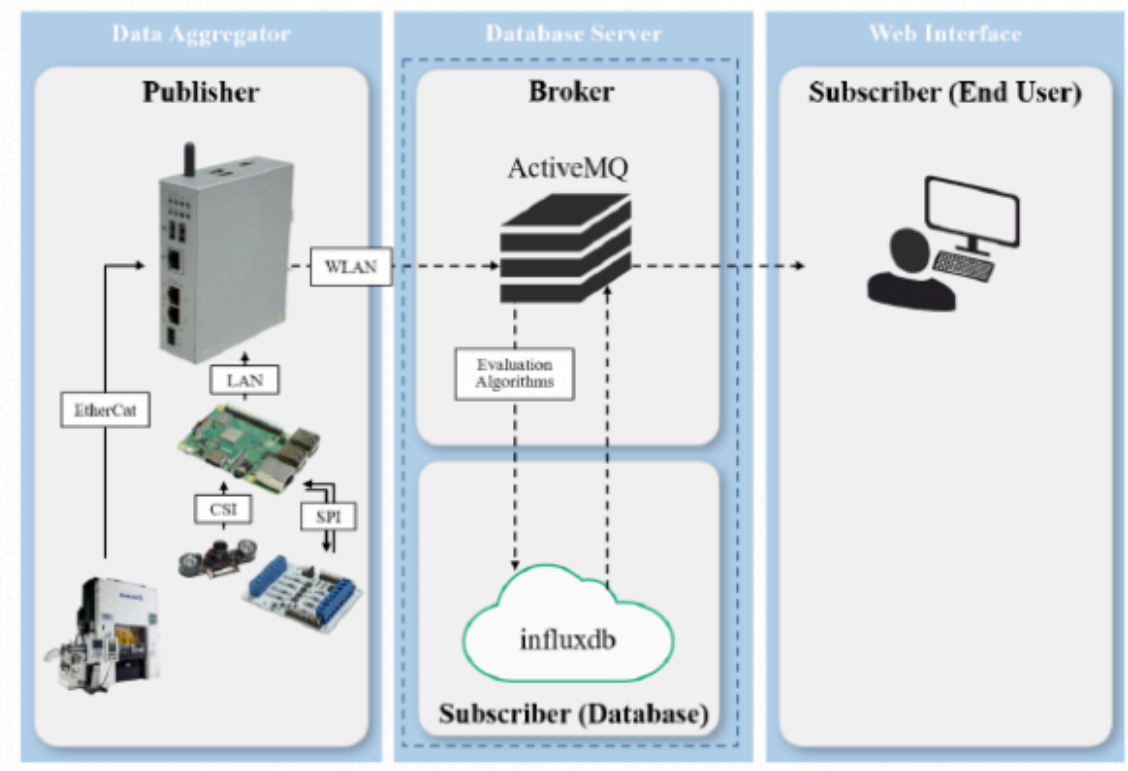

Fig. 5: MQTT-Setup

\section{Performance Test}

In order to enable application in real time, the transmission has to fulfil certain requirements regarding speed. Above all, the delay between acquisition and deployment of the data in the database is crucial. 
To ensure real time application, performance tests have been carried out, in which a data string is sent from the Raspberry Pi. The sent data consist of a timestamp and random values to obtain a $1 \mathrm{kB}$ data string. To avoid synchronization problems, the data are sent and received by the same component. In accordance with MQTT, the Raspberry Pi is both, Publisher and Subscriber. The timestamp in the received data then is compared to the time of receipt. Thus, the difference in time represents the delay of the transmission. For a transmission of 10,000 messages, an average duration of about $140 \mathrm{~ms}$ was calculated. This leads to a data rate of approximately $58.5 \mathrm{kbit} / \mathrm{s}$. This represents a first approximation, but further investigations are necessary to be able to provide a prediction about the measurable bandwidth of input signals.

Nevertheless, the wireless retransmission to the Raspberry Pi is not required in the final setup, so an even higher data rate can be expected. Besides this, an optimization of the program code can shorten the runtime and thus increase the performance as well.

\section{Summary and Future Work}

In the paper presented, a data acquisition system is introduced that can potentially be used in many different manufacturing environments since it is not limited to a specific bus system and can be extended with further external sensors. The use of highly compatible components such as the netPI and the Raspberry Pi simplifies changing the configuration. A first performance test has shown that the system provides a sufficient data rate to a certain level.

Future work focuses on the application of the acquisition system in a forming press. For this purpose, sheet material prepared with ID-codes will be used in an exemplary forming process. In the press, external sensors such as a temperature sensor, a humidity sensor or a LASER distance sensor are applied. Within this scope, further performance tests are also carried out. Moreover, the transmission security of process data also has to be evaluated, as it may be relevant for competition.

\section{Acknowledgements}

The Federal Ministry of Economics supports the IGF-project 20447 N of the European Research Association for Sheet Metal Working (EFB e.V.) via the German Federation of Industrial Research Associations (AiF) within the framework of the Industrial Collective Research (IGF) program and Technology based on a decision by the German Bundestag.

\section{Bibliography}

[1] Kaur, J. Arora, V. Bali, S. Influence of technological advances and change in marketing strategies using analytics in retail industry. International Journal of System Assurance Engineering and Management, 11, 2020, pp. 953-961. DOI 10.1007/s13198-020-01023-5

[2] Deloitte $\mathrm{GmbH}$, Predictive maintenance: Taking pro-active measures based on advanced data analytics to predict and avoid machine failure. Deloitte Analytics Institute Position Paper No. 7, 2017

[3] Purr, S. Moelzl, K. Meinhardt, J. Merklein, M. Das Presswerk 4.0 - Data Mining zur Vermeidung von Qualitätsproblemen bei der Herstellung von Karosseriebauteilen, Proceedings European Research Association for Sheet Metal Working Conference: Prozesstechnik in der Blechverarbeitung - Interaktion Maschine | Werkzeug, 2016 pp. 149-162, ISBN 978-3-86776-477-3

[4] Jatscha, A. Finus, F. Maschinenbau-Studie zur Zukunftssicherheit des Maschinenbaus. Maschinenmarkt, 09.07.2020

[5] Culic, I. Radovici, A. Rusu, C. Commercial and Industrial Internet of Things Applications with the Raspberry Pi. 


\section{A Versatile IoT-Approach to Process Data Acquisition}

Berkeley, CA: Apress, 2020. DOI 10.1007/978-1-4842-5296-3

[6] Matthias, S. Loderer, A. Koch, S. Gröne, M. Kästner, M. Hübner, S. Krimm, R. Reithmeier, E. Hausotte, T. Behrens, B.-A. Metrological solutions for an adapted inspection of parts and tools of a sheet-bulk metal forming process. Production Engineering Research and Development 10, 2016, pp. 51-61. DOI 10.1007/s11740-015-0647-2

[7] Carlsson, T. News From HMS: Industrial network market shares 2020 according to HMS Networks. 2020, https://www.hms-networks.com/news-and-insights/news-from-hms/2020/05/29/industrialnetwork-market-shares-2020-according-to-hms-networks, 27.11.2020

[8] Modular data acquisition system (DAQ) for any case: KiDAQ. 2020, https://www.kistler.com/en/applications/ sensor-technology/test-measurement/kidaq/, 05.10.2020

[9] CompactDAQ Systems. 2020, https://www.ni.com/en-us/shop/compactdaq.html, 05.10.2020

[10] ip4Cloud - The Clever IoT Controller. 2017, https://www.ipcomm.de/product/ip4Cloud/en/ ip4Cloud_Flyer_EN_V1.0.pdf, 05.10.2020

[11] netPI 3 RTE Industrial Raspberry Pi IoT Gateway "netPI RTE 3". 2020, https://www.hilscher.com/products/ product-groups/industrial-internet-industry-40/netiotnetfield-edge/niot-e-npi3-51-en-reecs/, 05.10.2020

[12] KUNBUS GmbH. Revolution Pi Flyer - Revolution Today. 2020

[13] PROFIBUS \& PROFINET International (PI). PROFINET Technology - The Easy Way to PROFINET. 2018

[14] PROFIBUS \& PROFINET International (PI). PROFIBUS System Description - Technology and Application. 2016

[15] CAN FD - The basic idea. 2020, https://www.can-cia.org/can-knowledge/can/can-fd/, 04.11.2020

[16] Arduino Yùn Rev.2 - Tech Specs. 2018, https://store.arduino.cc/arduino-yun-rev-2/, 05.10.2020

[17] Raspberry Pi 3 Model B+ - Specifications. 2018, https://www.raspberrypi.org/products/raspberry-pi-3-model-bplus/, 05.10.2020

[18] Behrens, B.-A. Vahed, N. Kammler, M. Functionalisation of PM components by integration of inherent data carriers and sensory elements. Production Engineering Research and Development 7, 2013, pp. 123-129. D0I 10.1007/ s11740-012-0428-0

[19] MQTT 5 Specification. 2019, https://docs.oasis-open.org/mqtt/mqtt/v5.0/mqtt-v5.0.html, 04.11.2020

[20] Pi-SPi-8AI+ Raspberry Pi Analog Input (4 - 20 mA) Interface. 2020, https://widgetlords.com/collections/pi-spiseries-1/products/pi-spi-8aiplus-raspberry-pi-analog-input-4-20-ma-interface, 05.10.2020

[21] RPi IR-CUT Camera, Better Image in Both Day and Night - Description: Features. 2020, https://www.waveshare.com/ product/rpi-ir-cut-camera.htm, 11.11.2020

PDF automatically generated on 2021-05-23 07:21:12

Article url: https://popups.uliege.be/esaform21/index.php?id=2043

published by ULiège Library in Open Access under the terms and conditions of the CC-BY License

(https://creativecommons.org/licenses/by/4.0) 